\title{
THE X-RAY BACKGROUND AND THE AGN LUMINOSITY FUNCTION
}

\author{
G. HASINGER \\ Astrophysikalisches Institut Potsdam \\ An der Sternwarte 16 \\ 14482 Potsdam \\ Germany
}

\begin{abstract}
ROSAT deep and shallow surveys have provided an almost complete inventory of the constituents of the soft X-ray background which led to a population synthesis model for the whole X-ray background with interesting cosmological consequences. According to this model the X-ray background is the "echo" of mass accretion onto supermassive black holes, integrated over cosmic time. A new determination of the soft X-ray luminosity function of active galactic nuclei (AGN) is consistent with pure density evolution, and the comoving volume density of AGN at redshift 2-3 approaches that of local normal galaxies. This indicates that many larger galaxies contain black holes and it is likely that the bulk of the black holes was produced before most of the stars in the universe. However, only Xray surveys in the harder energy bands, where the maximum of the energy density of the X-ray background resides, will provide the acid test of this picture.
\end{abstract}

\section{Introduction}

The soft X-ray background (XRB) has practically been resolved by deep ROSAT pencil beam survey observations into discrete sources and - at the faintest fluxes - source fluctuations (Hasinger et al., 1993; Branduardi et al., 1994). The ultradeep ROSAT HRI survey now reaches a surface density of $\sim 1000 \mathrm{deg}^{-2}$ at a flux of $10^{-15} \mathrm{erg} \mathrm{cm}^{-2} \mathrm{~s}^{-1}$ (Hasinger et al., 1998a). Counterparts of the weakest X-ray sources are optically very faint $(R<24)$ and require very good, unconfused X-ray positions and highquality optical spectra. The large majority of optical counterparts in the 
ROSAT deep surveys turned out to be AGN (Schmidt et al., 1998). X-ray selection is therefore the most efficient means to construct large, almost unbiased samples of distant AGN.

At the faintest $\mathrm{X}$-ray fluxes there is still a debate about the existence of a possible new population of nearby X-ray active, but optically innocent narrow emission line galaxies (NELG), whose X-ray luminosity may be powered by star forming processes (e.g. Griffiths et al., 1996; McHardy et al., 1997). These findings are, however, challenged by the higher resolution data in the Lockman Hole (Schmidt et al., 1998). For a more detailed review see Hasinger 1996.

\section{The ROSAT Ultra-Deep Survey}

An area of $\sim 0.3 \mathrm{deg}^{2}$ in the Lockman Hole was chosen as the field for the deepest X-ray survey ever, because it provides a minimum of interstellar absorption. Over the last 10 years it has become one of the best studied sky regions over a wide range of frequencies: UBVRI and K-band imaging from Kitt Peak, Mt. Palomar and Mauna Kea (Keck and UH), a 16hr VLA $20 \mathrm{~cm}$ mosaique (deRuiter et al., 1996), a 120ksec ASCA hard X-ray observation (Inoue et al., 1996) and deep IR mosaiques with ISOCAM and ISOPHOT aboard ISO (PIs: Cezarsky, Taniguchi) have been taken. The ROSAT Deep Survey (RDS) is a $207 \mathrm{ksec}$ PSPC exposure, and the ROSAT Ultradeep Survey an 1.11 Msec ROSAT HRI exposure in the Lockman Hole (Hasinger et al., 1998a).

The final limiting sensitivity for the detection of discrete sources is about $2 \times 10^{-15} \mathrm{erg} \mathrm{cm}^{-2} \mathrm{~s}^{-1}$ for the PSPC and about a factor of two fainter with the HRI. Figure 1 shows the PSPC and HRI $\log (\mathrm{N})-\log (\mathrm{S})$ function (Hasinger et al., 1998a) which is in very good agreement with data published previously. The HRI source counts reach a surface density of $970 \pm 150 \mathrm{deg}^{-2}$, about a factor of two higher than any previous X-ray determination. About $70-80 \%$ of the $\mathrm{X}$-ray background measured in the 1-2 $\mathrm{keV}$ band has been resolved into discrete sources now.

\section{Optical identifications}

Early optical identification programs concentrated on medium-deep ROSAT PSPC observations in AAT deep optical QSO fields and could quickly identify an impressive fraction of faint X-ray sources as classical broad-line AGNs (mainly QSOs) (e.g. Shanks et al., 1991, Georgantopoulos et al., 1996). There was, however, mounting evidence that a new class of sources might start to contribute to the XRB at faint X-ray fluxes. The faintest $\mathrm{X}$-ray sources in ROSAT deep surveys on average show a harder spectrum than the identified QSOs (Hasinger et al., 1993). In medium-deep pointings 


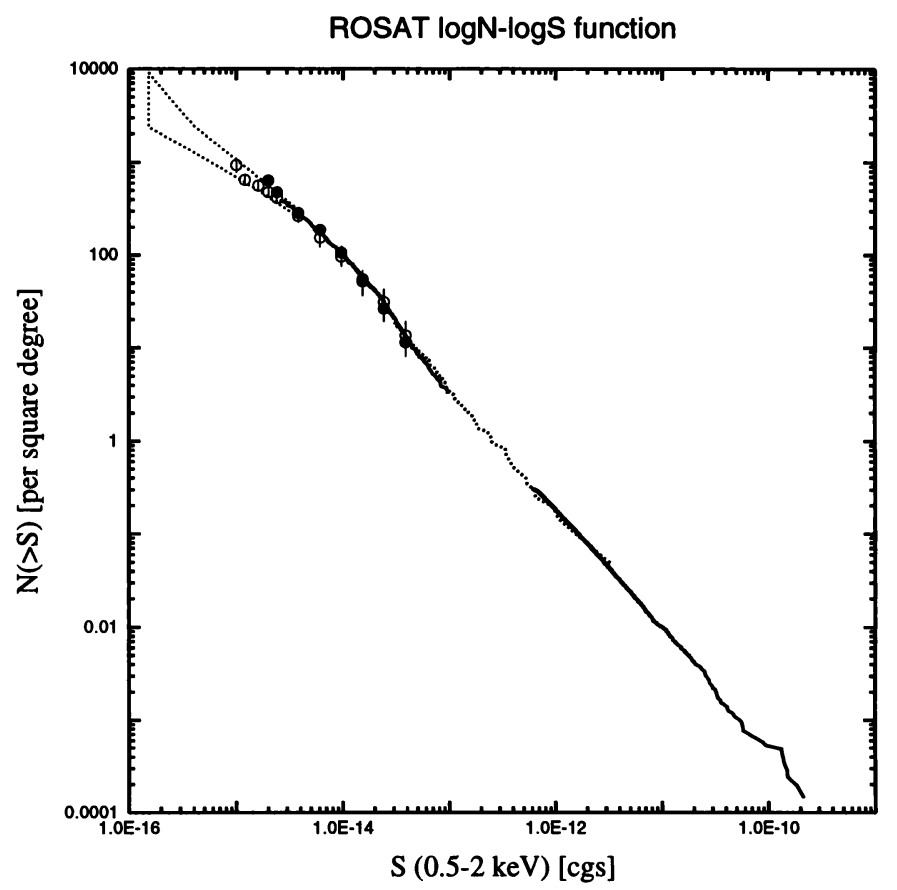

Figure 1. Total $\log (\mathrm{N})-\log (\mathrm{S})$ function determined from various ROSAT surveys. Filled circles give the source counts from the 207 ksec PSPC observation in the Lockman Hole (Hasinger et al., 1998a). Open circles are from the HRI ultradeep survey observation $(1.112 \mathrm{Msec})$. The data are plotted on top of the source counts (solid line) and fluctuation limits (dotted area) from Hasinger et al. (1993). The dotted line at intermediate fluxes refers to the total source counts from the RIXOS survey (Mason et al., 1996). The solid line at bright fluxes was determined from the ROSAT All-Sky Survey bright source catalogue (Voges et al., 1995).

a number of optically "innocent" narrow-emission line galaxies (NELGs) at moderate redshifts $(\mathrm{z}<0.4)$ were identified as $\mathrm{X}$-ray sources, which was in excess of those expected from spurious identifications with field galaxies (Boyle et al., 1995, Georgantopoulos et al., 1995, Griffiths et al., 1996). Roche et al.(1995) have found a significant correlation of X-ray fluctuations with optically faint galaxies. Finally, in an attempt to push optical identifications to the so far faintest X-ray fluxes, McHardy et al. (1997) claim that the surface density of broad-line AGN flattens dramatically at

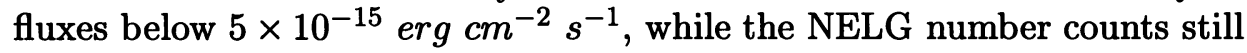
keep increasing, so that those would eventually dominate the XRB below a flux of $10^{-15} \mathrm{erg} \mathrm{cm}^{-2} \mathrm{~s}^{-1}$.

While this is obviously an interesting possibility, it is useful to remind that all these findings are based on identifications near the limit of deep PSPC surveys, at fluxes where our simulations suggest that the PSPC 


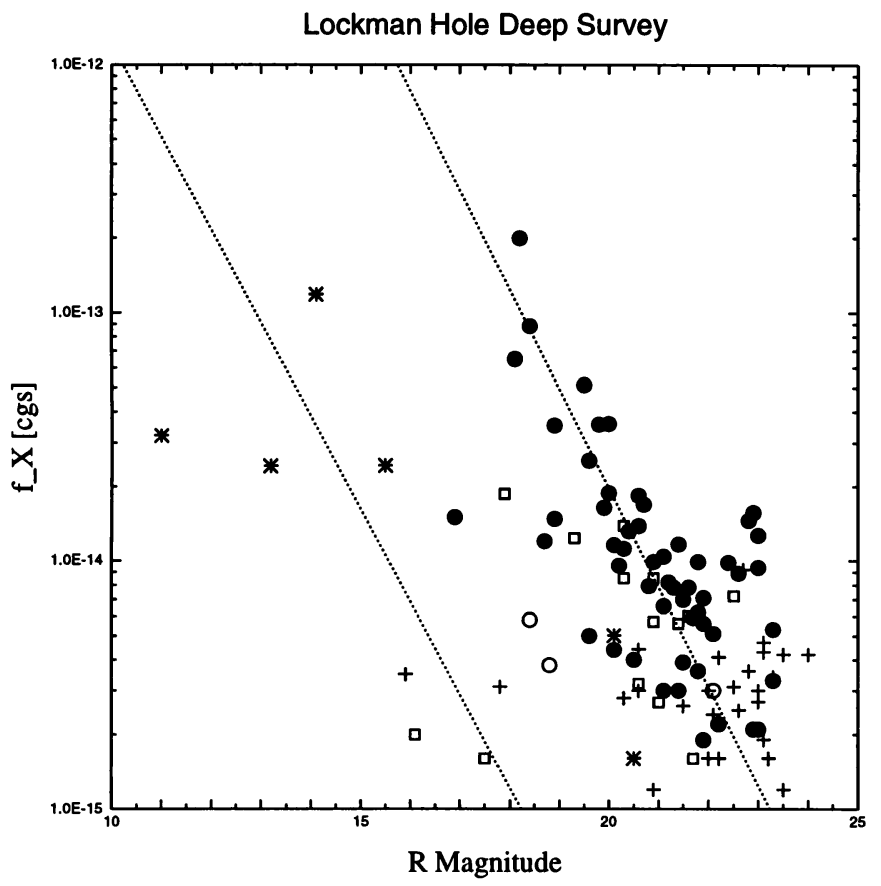

Figure 2. Correlation between X-ray flux and optical $\mathrm{R}$ magnitude for all objects in the Lockman Hole survey. Filled circles give spectroscopically identified AGN, open circles unspecified galaxies, open squares candidate clusters and group of galaxies. Asterisks show coronal stars and plus signs unidentified objects, in this case always the brightest optical counterpart in the $90 \%$ error circle is shown. The catalogue of sources down to X-ray fluxes of $5.5 \times 10^{-15}$ has been published by Schmidt et al., 1998. Fainter objects are subject of the ongoing multislit spectroscopy and therefore provides a fair sample of the data.

data start to be severely confused, with some likelihood of misidentification (Hasinger et al., 1998a). Also, spectroscopic optical identifications in these samples are limited to $R<22$.

In the Lockman Hole Deep survey, optical counterparts have magnitudes in the range $R=19-24$. With the excellent HRI positions (of order 2-4 arcsec) typically only one counterpart is within the X-ray error box. In Fig. 2 the Xray fluxes of our sources are plotted against the magnitude of their optical counterparts or, in case of no identification, the brightest optical candidate in the error box. Long-slit and multislit spectra of the 1-3 candidates closest to the X-ray source have been taken using the Palomar 200" 4-shooter and the Keck LRIS instruments. Reliable spectroscopic identifications are now practically complete in a $0.30 \mathrm{deg}^{2}$ field to a flux limit of $1.1 \cdot 10^{-14} \mathrm{cgs}$ and in the central $0.14 \mathrm{deg}^{2}$ of the field down to a flux limit of $5.5 \cdot 10^{-15} \mathrm{cgs}$ 
(Schmidt et al., 1998) and in a smaller area, which we continue to work on, down to $10^{-15} \mathrm{cgs}$. The majority of the optical counterparts are active galactic nuclei with broad emission lines (QSOs and Seyfert galaxies) in the redshift range $0.08-4.5$, whereas only a very small fraction of non-AGN NELGs is found. Data on the highest redshift X-ray selected QSO will be presented elsewhere (Schneider et al., 1998). A large fraction of the faint X-ray sources are optically resolved low luminosity AGN (Seyfert galaxies), some of which show clear evidence of gas and dust obscuration in the X-ray and optical band (i.e. Seyfert 1.5-2 galaxies), which are very hard to select by any other means than X-rays.

The data of McHardy et al. (1997) is roughly consistent with our findings, if we consider three selection/incompleteness effects, which are partly discussed in their work: a.) their optical identifications are restricted to $R<22$, while we have optical counterparts as faint as $\mathrm{R}=24$. b.) they call some fraction of objects NELGs, which due to our higher quality spectra reveal some evidence for AGN activity, and c.) some fraction of their NELGs may be misidentified due to source confusion.

\section{A new AGN X-ray luminosity function (XLF)}

Using data from medium deep ROSAT fields combined with the Einstein Medium Sensitivity Survey (Gioia et al., 1990), Boyle et al. (1994) could derive the AGN XLF and its cosmological evolution. Their data is consistent with pure luminosity evolution proportional to $(1+z)^{2.7}$ up to a redshift $z_{\max } \approx 1.5$, similar to what was found previously in the optical range. This result has been confirmed and improved later on by more extensive or deeper studies of the AGN XLF, e.g. the RIXOS project (Page et al., 1996) or the UK deep survey project (Jones et al., 1996). All these studies agree that at most half of the faint $\mathrm{X}$-ray source counts can be explained by classical broad-line AGN based on the pure luminosity evolution models. However, the limited information at bright X-ray fluxes and in particular the uncertain crosscalibration between the ROSAT and Einstein surveys severely limits the accuracy of the AGN XLF (Boyle et al., 1994; Page et al., 1996).

We can now determine a new AGN soft X-ray luminosity function based on ROSAT surveys alone and covering a very wide range of limiting fluxes. For this purpose the deep survey sample described above has been combined with optical identifications from shallower wide angle surveys, i.e. the ROSAT Bright Survey (RBS, Hasinger et al., 1998b), derived from the ROSAT All-Sky Survey bright source catalogue (Voges et al., 1995), and the RIXOS AGN sample (Page et al., 1996). The accuracy of the relative flux scale for these surveys is demonstrated by the $\log \mathrm{N}-\log \mathrm{S}$ function in Fig. 1, 


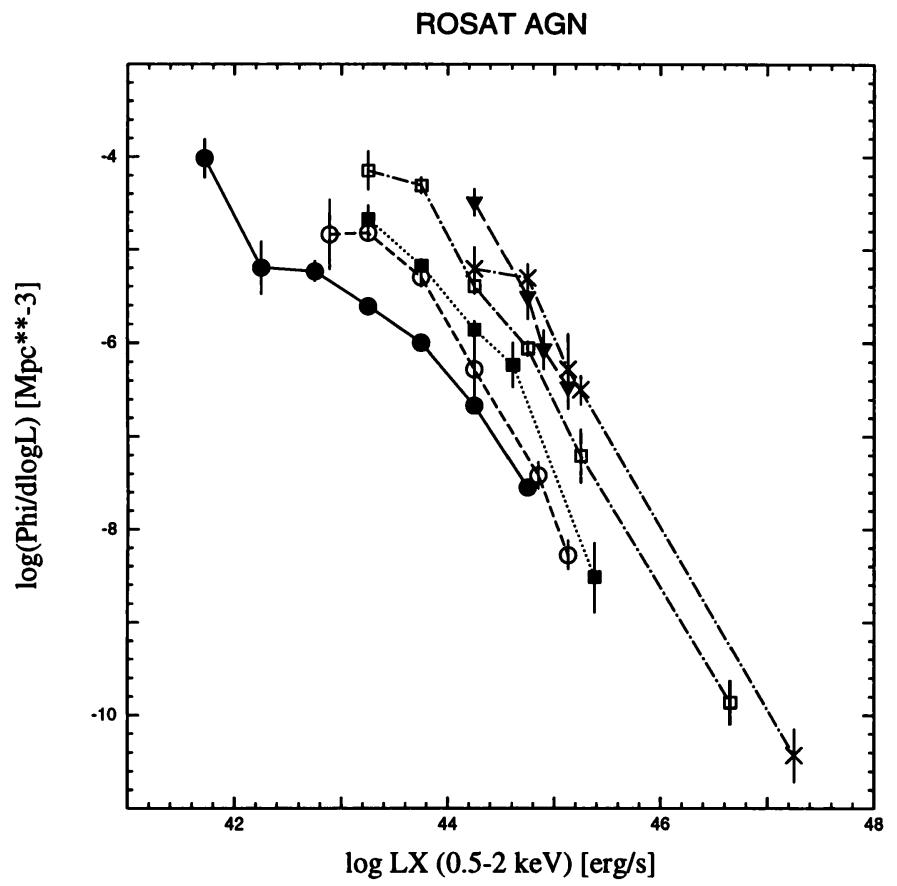

Figure 3. The AGN X-ray luminosity function derived from a joint analysis of the ROSAT Deep and Ultradeep Survey, RIXOS and the ROSAT Bright Survey. The comoving AGN volume density is displayed as a bivariate function of luminosity and redshift. Redshift shells are 0-0.2 (filled circles), 0.2-0.4 (open circles), 0.4-0.8 (filled squares), 0.8-1.6 (open squares), 1.6-2 (filled triangles), and 2-4.5 (asterisks).

where the different surveys agree within $\sim 10 \%$ in the overlap regions over a flux range of about six orders of magnitude.

Fig. 3 shows the binned AGN XLF in different redshift shells. Consistent with Boyle et al., we find strong cosmological evolution in the sense that high-redshift AGN are much more abundant or more luminous than their local counterparts. Contrary to the Boyle et al. findings, however, the new XLF is not consistent with pure luminosity evolution. For the first time we see evidence for strong cosmological evolution of the space density of low-luminosity AGN (e.g. Seyfert galaxy) XLF out to a redshift 1-2, incompatible with pure luminosity evolution. Surprisingly, however, a pure density evolution model of the form $\Phi(z)=\Phi(0) \cdot(1+z)^{5}$ can fit the data well.

Fig. 4 shows the luminosity function, de-evolved under the assumption of pure density evolution. A smoothed broken power law model of the form

$$
\Phi(L, z) / \operatorname{dlog} L=(1+z)^{5.03} \frac{2 \cdot 10^{-6}}{l^{2.03}+l^{0.51}}\left[M p c^{-3}\right]
$$




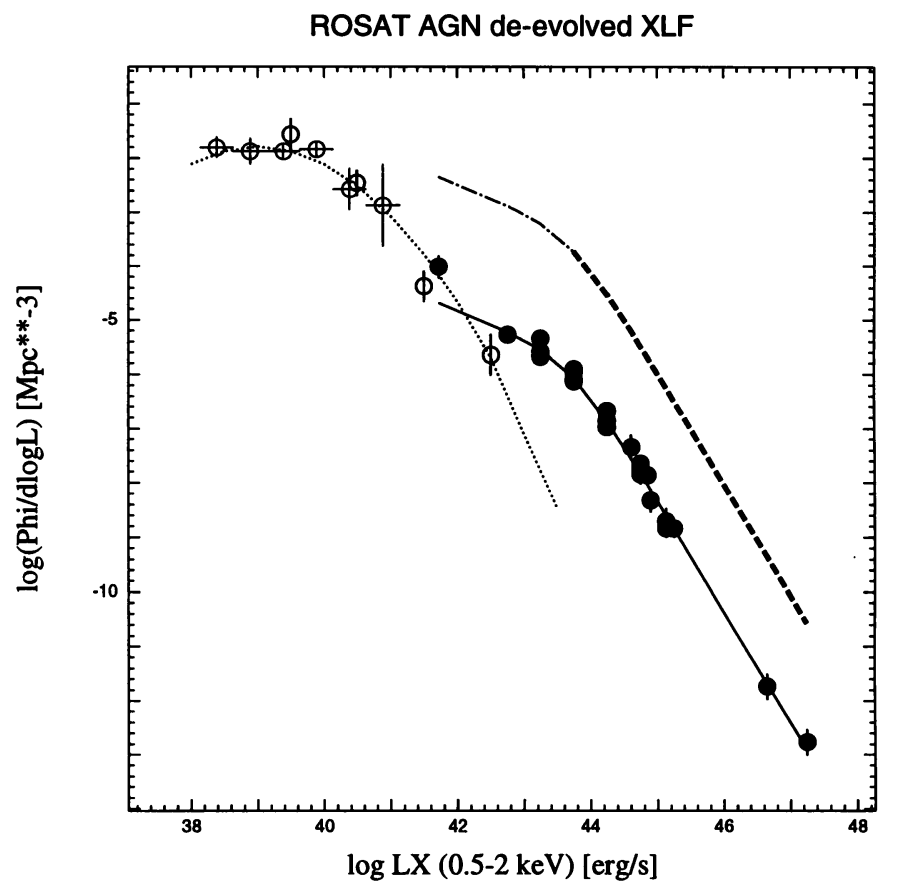

Figure 4. X-ray Luminosity functions for AGN and galaxies from ROSAT surveys. The local galaxy luminosity function (open circles) has been derived from the ROSAT Bright Survey (Hasinger et al., 1998b) and from a volume-limited sample of local galaxies (Schmidt, Boller and Voges, 1996). A log-normal distribution has been fit to the galaxy XLF (dotted curve). The AGN luminosity function (filled circles) has been de-evolved using a pure density evolution model (see text). A smoothed broken power law model (solid line) has been fit to the data. The dashed curve indicates the model AGN luminosity function observed in the redshift range 2-3, its dash-dotted extension to lower luminosities is the model based on pure density evolution (see text).

has been fit to the de-evolved XLF out to redshifts of 1.92. Here $l=$ $L_{X} / 5 \cdot 10^{43}$. The model for $\mathrm{z}=0$ is shown in figure 4 by a solid line. The model for the $\mathrm{z}=2 \mathrm{XLF}$ over the observed luminosity range is shown by the thick dashed line, its extrapolation to lower luminosities by the dash-dotted line.

Integrating the luminosity function to $z \approx 2$ we we actually overpredict the soft X-ray background flux and the soft X-ray logN-logS function and fluctuations. One has to stress however, that this is only a first-order treatment of the data. The effect of intrinsic X-ray absorption and the detailed shape of the AGN spectra has to be included in the derivation of the XLF in order to obtain a self-consistent population synthesis model for the XRB spectrum (see Comastri et al., 1995). Also, the so far unobserved low 
luminosity range of the high-redshift AGN XLF provides a significant contribution to the XRB and the faint number counts and is therefore crucial in understanding the composition of the XRB. Even deeper observations, preferrably at higher X-ray energies will thus be necessary.

\section{Does every galaxy contain a black hole?}

This question has originally been posed by Rees (1989). With recent data from HST and from the deep X-ray surveys we can now shed new light on this question. In fig. 5 we plot the volume density evolution of X-ray selected AGN (this work) and optically selected AGN as a function of redshift (Schmidt, Schneider \& Gunn 1995). The AGN space density shows a large variation with redshift (a factor of several hundred) with a marked peak at $\mathrm{z}=2-3$. Note that only the AGN space density derived from a pure density evolution model shows such a large variation against cosmic time, while the volume luminosity derived from a pure luminosity evolution model shows a much smaller variation (see e.g. Boyle and Terlevich, 1997). The extrapolated comoving volume density of X-ray selected QSO and Seyfert galaxies at $\mathrm{z}=2-3$, albeit the systematic uncertainties discussed above, approaches that of the low-redshift normal galaxies (see Fig. 4) as well as that of the recently discovered population of Lyman-limit galaxies at high redshifts (Steidel et al., 1996). This indicates that a substantial fraction of normal galaxies may host a central supermassive black hole, which turns into an AGN as soon as it is fuelled, e.g. by interactions. The amount of interactions is much larger at higher redshifts than today, which can explain the sharp drop of activity towards low z. Dormant remnants of AGN are indeed found in almost every nearby galaxy with a spheroidal component. A tight relation between black hole mass and bulge mass has been found, indicating that there is a causal connection between the size of the central black hole and the number of stars in a galaxy (Faber et al., 1996).

Recently the history of global star formation in the universe been determined e.g. through observations in the Hubble deep field, but also from the chemical evolution of $\mathrm{Ly}_{\alpha}$ clouds. Fig. 5 gives the starforming rate in arbitrary units as a function of redshift (Madau 1996, Conolly et al., 1997), corrected for dust obscuration following Pettini et al. (1997). This function shows only a moderate variation with cosmic time, with a possible peak around a redshift of unity and a steep decline towards lower redshifts, the shape of which is strikingly similar to the low-redshift behaviour of the AGN volume density.

The pronounced peak of the AGN population appears to be at higher redshift than the maximum of the star formation, indicating, that the bulk of black holes which nowadays form the center of normal galaxies has been 


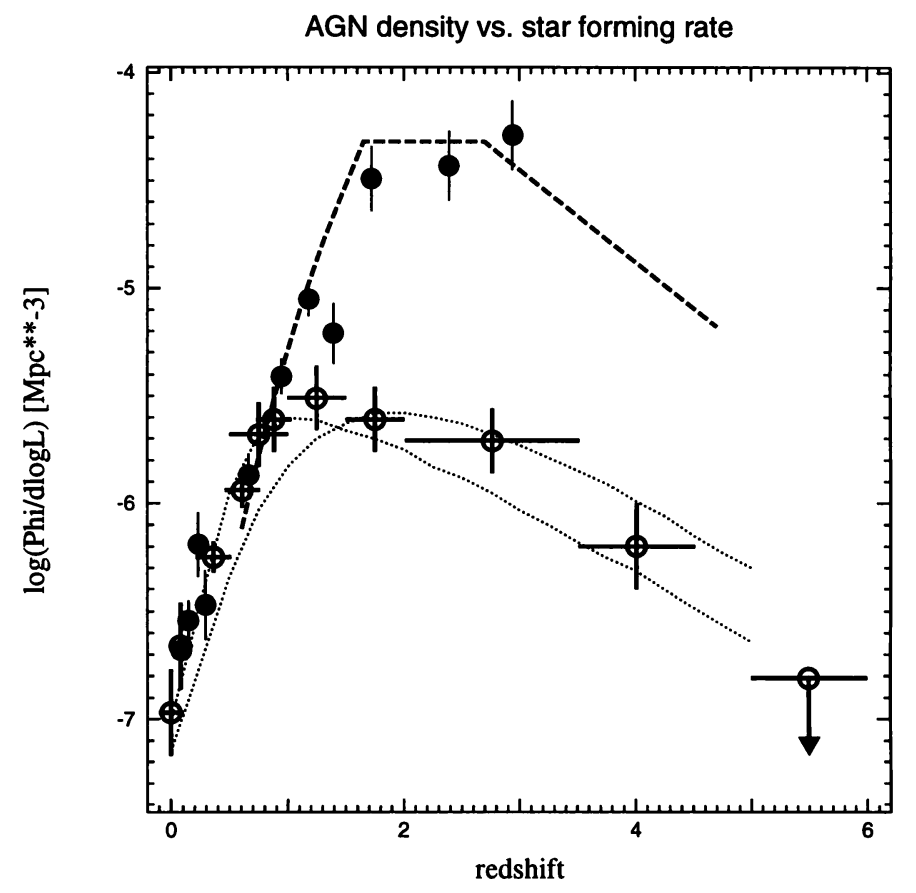

Figure 5. The filled circles give the volume density of X-ray selected AGN referred to an X-ray luminosity of $\log L_{X}=44.25$. The dashed curve sketches the volume density of optically selected QSO with $M_{B}<-26$ (Schmidt, Schneider and Gunn, 1995), increased by a factor of 250 to account for the different luminosities in the different wavebands. The open circles show the star formation rate as a function of redshift from Madau 1996 and Connolly et al., 1997, corrected for dust obscuration (Pettini et al., 1997). The dotted curves give models for the star formation rate derived from the chemical evolution of $H_{\alpha}$ clouds (Pei and Fall 1995). The latter two curves are in arbitrary units, scaled to the AGN density at low redshifts.

in place before the bulk of the stars in these galaxies have formed. In this picture the X-ray background is just the total emission of all black hole accretion processes, integrated over cosmic space and time. Following the argument by Soltan (1982), the total mass accreted onto black holes integrated over cosmic time can be estimated from the AGN number counts and thus from the X-ray background radiation. This corresponds to an average mass in dormant black holes of $10^{7}-10^{8} M_{\odot}$ per galaxy (see e.g. Chokshi \& Turner 1992) and is surprisingly close to the average black hole mass measured for local galaxies (Faber et al., 1996).

The scenario described here suggests an attractive solution to the $\mathrm{X}$ ray background puzzle, where the XRB is just the "echo" of mass accretion processes onto supermassive black holes in the centers of nearly all galaxies. However, only deeper survey observations with the next generation of sen- 
sitive X-ray observatories with good angular resolution in the harder X-ray band (i.e. AXAF and XMM), where the bulk of the energy density of the $\mathrm{XRB}$ resides, and optical follow-up spectroscopy from 8-10m telescopes will be able to unambiguously confirm the AGN XRB model and finally solve this long-standing mystery.

\section{Acknowledgements}

I acknowledge the grant 50 OR 94035 by the Deutsche Agentur für Raumfahrtangelegenheiten (DARA). I also warmly thank my collaborators in the ROSAT Deep Survey project, Riccardo Giacconi, Maarten Schmidt, Joachim Trümper and Gianni Zamorani for fruitful discussions and the permission to discuss data in advance of publication. In particular I am grateful to M. Schmidt for help with the luminosity function.

\section{References}

Boyle B.J. et al., 1994, MNRAS 260, 49

Boyle B.J. et al., 1995, MNRAS 272, 462

Boyle B.J., Terlevich R.J., 1998, MNRAS submitted (astro-ph/9710134)

Branduardi-Raymont G. et al., 1994, MNRAS 270, 947

de Ruiter H. et al., 1997, A\&A 319, 7

Faber S.M. et al., 1997, AJ in press (astro-ph/9610055)

Georgantopoulos I. et al., 1996, MNRAS 280, 276

Gioia I.M. et al., 1990, ApJ Suppl. 72, 567

Griffiths R.E. et al., 1996, MNRAS 281, 71

Hasinger G. 1996, A\&A Suppl. 120, C607

Hasinger G. et al., 1993, A\&A 275, 1

Hasinger et al., 1998a, A\&A, in press (astro-ph/9709142)

Hasinger et al., 1998b, AN, in prep.

Inoue H. 1996, MPE report 263, 323

Jones L.R. et al., 1996, MNRAS, submitted, (astro-ph/9610124)

Madau P., 1996, astro-ph 9612157

Mason, K. et al., 1996, priv. comm

McHardy I.M. et al., 1997, MNRAS, submitted, (astro-ph/9703163)

Page M.J. et al., 1996, MNRAS 281, 579

Pettini M., et al., 1997, astro-ph 9708117

Rees M., 1989, Reviews of Modern Astronomy 2,1

Roche N. et al., 1995, MNRAS 273, 15

Schmidt K.-H., Boller T., Voges W., MPE report 263, 395

Schmidt M., Schneider D.P., Gunn J.E., 1995, AJ 110, 68

Schmidt M. et al. 1998, A\&A, in press (astro-ph/9709144)

Schneider D., et al., 1998, AJ submitted

Shanks T. et al. 1991, Nature 353, 315

Steidel, C. et al., 1996, AJ 112, 352

Voges, W., et al., 1995, IAU Circ \# 6102 